CU-TP-540

FERMILAB-Pub-91/326-A\&T

\title{
A Classical Instability of Reissner-Nordström Solutions and the Fate of Magnetically Charged Black Holes
}

\author{
Kimyeong Lee, ${ }^{a *}$ V.P.Nair ${ }^{a}$, and Erick J. Weinberg ${ }^{a, b}$ \\ ${ }^{a}$ Physics Department, Columbia University \\ New York, New York 10027 \\ ${ }^{b}$ Theory Group and NASA/Fermilab Astrophysics Center \\ Fermi National Accelerator Laboratory \\ P.O.Box 500, Batavia, Illinois 60510.
}

\begin{abstract}
Working in the context of spontaneously broken gauge theories, we show that the magnetically charged Reissner-Nordström solution develops a classical instability if the horizon is sufficiently small. This instability has significant implications for the evolution of a magnetically charged black hole. In particular, it leads to the possibility that such a hole could evaporate completely, leaving in its place a nonsingular magnetic monopole.
\end{abstract}

This work was supported in part by the US Department of Energy (VPN and EJW), by NASA (EJW) under grant NAGW-2381 and by an NSF Presidential Young Investigator Award (KL).

* Alfred P. Sloan Fellow. 
The Reissner-Nordström solution to the coupled Einstein-Maxwell equations describes a spherically symmetric black hole endowed with electric or magnetic charge. Although the solution makes mathematical sense in a theory involving only gravity and the electromagnetic field, its physical motivation is somewhat tenuous unless the theory also contains particles carrying such charges. This remark has little consequence in the case of electric charge, since one only need add a field whose elementary particles are electrically charged. The addition of magnetic charge to the theory is less trivial, but can be accomplished by enlarging the structure of the theory so that electromagnetism emerges as the unbroken subgroup of a spontaneously broken gauge theory in which magnetic monopoles arise as topologically nontrivial classical solution [1]. The incorporation of these additional fields into the Reissner-Nordström solution is rather straightforward [2], and changes neither the metric nor the magnetic field. However, as we will show in this letter, the presence of these fields can render this solution unstable. This instability arises at the level of the classical field equations and does not depend on any quantum mechanical process. It has important implications for the ultimate fate of magnetically charged black holes.

The magnetically charged Reissner-Nordström solution to the Maxwell-Einstein equations has a radial magnetic field with magnitude $Q_{M} / r^{2}$ and a metric which may be written as

$$
d s^{2}=B d t^{2}-A d r^{2}-r^{2} d \theta^{2}-r^{2} \sin ^{2} \theta d \phi^{2}
$$

where

$$
B=A^{-1}=1-\frac{2 M G}{r}+\frac{4 \pi G Q_{M}^{2}}{r^{2}} \equiv B_{R N}
$$

There is a physical singularity at $r=0$ which is hidden within a horizon at

$$
r_{H}=M G+\sqrt{M^{2} G^{2}-4 \pi G Q_{M}^{2}}
$$

provided that the mass $M$ is greater than

$$
M_{c r i t}=\sqrt{4 \pi}\left|Q_{M}\right| M_{P}
$$

where the Planck mass $M_{P}=G^{-1 / 2}$. If $\left|Q_{M}\right| \gg 1$ (as will be the case for the weak gauge coupling we will assume) the horizon of the critical Reissner-Nordström black hole is at $r_{H} \gg M_{P}^{-1}$, and is thus in a region where quantum gravity effects can be neglected. 
This solution is readily incorporated into a theory possessing classical magnetic monopole solutions. For definiteness we consider an $S U(2)$ gauge theory which is spontaneously broken to $U(1)$ by the vacuum expectation value of a triplet Higgs field $\boldsymbol{\Phi}$. The action is

$$
S=\int d^{4} x \sqrt{-g}\left[-\frac{1}{16 \pi G} R+\mathcal{L}_{\text {Matter }}\right]
$$

where

$$
\begin{gathered}
\mathcal{L}_{\text {Matter }}=-\frac{1}{4} \mathbf{F}_{\mu \nu} \cdot \mathbf{F}^{\mu \nu}+\frac{1}{2} D_{\mu} \mathbf{\Phi} \cdot D^{\mu} \mathbf{\Phi}-V(|\boldsymbol{\Phi}|) \\
\mathbf{F}_{\mu \nu}=\partial_{\mu} \mathbf{A}_{\nu}-\partial_{\nu} \mathbf{A}_{\mu}-e \mathbf{A}_{\mu} \times \mathbf{A}_{\nu} \\
D_{\mu} \mathbf{\Phi}=\partial_{\mu} \mathbf{\Phi}-e \mathbf{A}_{\mu} \times \boldsymbol{\Phi}
\end{gathered}
$$

and vector notation refers to the internal $S U(2)$ indices. The potential $V(|\boldsymbol{\Phi}|)$ is assumed to have a minimum at $|\mathbf{\Phi}|=v$; to avoid a cosmological constant, $V$ must vanish at this minimum. This theory contains nonsingular monopoles with magnetic charge $Q_{M}=1 / e$ and mass $M_{\text {mon }} \sim 4 \pi v / e$, provided that $v \lesssim M_{P}$. (For $v$ larger than this, the would-be monopoles are so massive that they become black holes themselves $[3,4]$.)

The metric for the Reissner-Nordström solutions of this theory is precisely the same as that given above for the Maxwell theory. For vanishing electric charge and magnetic charge $Q_{M}=n / e$, the matter fields are, up to a possible gauge transformation,

$$
\begin{aligned}
\mathbf{\Phi} & =v \hat{\mathbf{e}}(\theta, \phi) \\
\mathbf{A}_{\mu} & =\frac{1}{e} \hat{\mathbf{e}} \times \partial_{\mu} \hat{\mathbf{e}}
\end{aligned}
$$

where $\hat{\mathbf{e}}$ is a unit vector with winding number $n$; a convenient choice, which we adopt henceforth, is $\hat{\mathbf{e}}=(\sin \theta \cos n \phi, \sin \theta \sin n \phi, \cos \theta)$. These imply that

$$
\mathbf{F}_{\theta \phi}=-\mathbf{F}_{\phi \theta}=\frac{n}{e} \sin \theta \hat{\mathbf{e}}
$$

This lies entirely within the electromagnetic $U(1)$ subgroup defined by the Higgs field and precisely reproduces the radial magnetic field of the Maxwell-Einstein theory. All other components of the field strength, as well as all of the covariant derivatives of $\boldsymbol{\Phi}$, vanish. 
We now investigate the stability of these solutions, beginning with the case of perturbations about the solution with unit magnetic charge. The problem can be simplified by considering only spherically symmetric configurations; this turns out to be sufficient to demonstrate instability. For such configurations, the metric can be written in the form of Eq.(1), with $B$ and $A$ being functions only of $r$ and $t$. By an appropriate gauge choice the matter fields can be brought to the form

$$
\begin{gathered}
\boldsymbol{\Phi}=v \hat{\mathbf{e}}(\theta, \phi) h(r, t) \\
\mathbf{A}_{i}=\frac{1}{e} \hat{\mathbf{e}} \times \partial_{i} \hat{\mathbf{e}}(1-u(r, t))
\end{gathered}
$$

where $A_{0}=0$ because we are interested in electrically neutral solutions. (With our choice for $\hat{\mathbf{e}}$, this reduces to the standard ansatz in flat space.) Substitution of this into the matter Lagrangian gives

$$
\mathcal{L}_{\text {Matter }}=\frac{1}{B}\left[\frac{\dot{u}^{2}}{e^{2} r^{2}}+\frac{1}{2} v^{2} \dot{h}^{2}\right]-\frac{1}{A}\left[\frac{u^{\prime 2}}{e^{2} r^{2}}+\frac{1}{2} v^{2} h^{\prime 2}\right]-\frac{\left(u^{2}-1\right)^{2}}{2 e^{2} r^{4}}-\frac{u^{2} h^{2} v^{2}}{r^{2}}-V(h)
$$

with overdots and primes referring to derivatives with respect to $t$ and $r$, respectively. This leads to the equations

$$
\frac{1}{\sqrt{A B}} \frac{\partial}{\partial t}\left(\frac{\sqrt{A B} h}{B}\right)-\frac{1}{r^{2} \sqrt{A B}} \frac{\partial}{\partial r}\left(\frac{r^{2} \sqrt{A B} h^{\prime}}{A}\right)=-\frac{2 h u^{2}}{r^{2}}-\frac{1}{v^{2}} \frac{d V}{d h}
$$

and

$$
\frac{1}{\sqrt{A B}} \frac{\partial}{\partial t}\left(\frac{\sqrt{A B} \dot{u}}{B}\right)-\frac{1}{\sqrt{A B}} \frac{\partial}{\partial r}\left(\frac{\sqrt{A B} u^{\prime}}{A}\right)=-\frac{u\left(u^{2}-1\right)}{r^{2}}-e^{2} u h^{2} v^{2}
$$

for the matter fields, as well as equations, whose explicit form we do not need, for the metric coefficients $A$ and $B$. To consider fluctuations about the Reissner-Nordström solution we only need keep terms linear in $u, h-1, \delta B \equiv B-B_{R N}$, and $\delta A \equiv A-B_{R N}^{-1}$. Remarkably, the coupled equations separate. The equations for the metric components contain neither $u$ nor $h-1$, and thus cannot lead to unstable modes (otherwise there would be an instability in the pure Maxwell-Einstein case). The perturbation of the scalar field enters only in the linearization of Eq. (15), and can be shown not to lead to instability. The remaining 
fluctuation, $u$, is determined by the linearized version of Eq. (16). If we define a variable $x$ by

$$
\frac{d x}{d r}=\frac{1}{B_{R N}(r)}
$$

so that $x$ ranges from $-\infty$ to $\infty$ as one goes from the horizon to spatial infinity, then the equation for $u$ may be written as

$$
0=\ddot{u}-\frac{d^{2} u}{d x^{2}}+U(x) u
$$

where

$$
U(x)=B_{R N}(r) \frac{\left(e^{2} v^{2} r^{2}-1\right)}{r^{2}}
$$

and $r$ is understood to be a function of $x$ determined by Eq. (17). Instability occurs if there are solutions of the form $u(r, t)=f(r) e^{\omega t}$ with real $\omega$. Substitution of this gives a one-dimensional Schroedinger equation for a particle moving under the influence of the potential $U(x)$. The unstable mode exists if this potential has a bound state. Since $U(x)$ goes to the positive value $e^{2} v^{2}$ at $x=\infty$, although it goes to zero at $x=-\infty$, it is not entirely trivial to see for what range of parameters we have a bound state. One can show that a bound state exists if $r_{H}<c(e v)^{-1}$ where $c$ is somewhat less than one, or, equivalently for

$$
M<\frac{c M_{P}^{2}}{2 e v}+\frac{2 \pi v}{c e}
$$

As $M \rightarrow M_{c r i t}, c$ approaches unity. For $M \gg M_{c r i t}$, we can bound $c$ by a variational calculation. Using the variational ansatz $u=\sqrt{r-r_{H}} \exp \left(-\lambda\left(r-r_{H}\right) / 2\right)$, we find $c>0.32$.

The physical basis for this instability is easily understood. The classical monopole solution has a core of radius $\sim(e v)^{-1}$, inside of which the Higgs field deviates from its vacuum value and the massive components of the gauge field are nonzero. The effect of this core is to remove the singularity in the energy density which would arise from a point magnetic charge. Its radius is determined by the balancing of the energy needed to produce the nontrivial matter fields against the energy cost of extending the Coulomb magnetic field further inward.

Similar considerations can be applied to solutions with horizons. Here, however, we should only consider the region outside the horizon since singularities are allowed, and 
even expected, inside the horizon. Looking at the case of a Reissner-Nordström solution with $r_{H} \lesssim(e v)^{-1}$, we see that the Coulomb field has, in a sense, been extended inward too far. Energetically, it would be preferable to have a core region extending outward beyond the horizon [5]. In fact solutions of this sort, which may be viewed as small black holes lying within larger magnetic monopoles, can be shown to exist if $v$ is less than a critical value $v_{c r} \sim M_{P}$ and if the mass $M$ is not too great [4]. When they exist, the horizon radius $r_{H}$ of these solutions is larger than that of the Reissner-Nordström solution with the same value of $M$. Thus, these solutions appear to be the natural endpoints to which the instability of the Reissner-Nordström solution leads.

We now turn to the case of multiple magnetic charge. The analysis is complicated by the fact that in the $S U(2)$ theory the only configurations with higher topological charge which are spherically symmetric (i.e., invariant up to a gauge transformation under spatial rotations) are the singular solutions given by Eqs. (9) and (10) [6]. There is thus no spherically symmetric case to which we can restrict our consideration; instead, we must consider the full perturbation problem. This can be done by expanding the action in powers of the fluctuations about the Reissner-Nordström solution and examining the terms quadratic in these fluctuations. (The linear terms vanish because we are expanding about a solution.) It is convenient to use the gauge freedom to require that the orientation of the scalar field remain the same as in the unperturbed solution, so that $\delta \boldsymbol{\Phi} \times \hat{\mathbf{e}}=0$. It is also useful to decompose the fluctuation in the gauge field into parts orthogonal to and parallel to $\hat{\mathbf{e}}$; thus, we write $\delta \mathbf{A}_{\mu}=\mathbf{a}_{\mu}+c_{\mu} \hat{\mathbf{e}}$ with $\hat{\mathbf{e}} \cdot \mathbf{a}_{\mu}=0$. The fact that $D_{\mu} \hat{\mathbf{e}}=0$ (here, and for the remainder of this discussion, $D_{\mu}$ is the covariant derivative defined by the unperturbed vector potential) leads to the useful result that $\hat{\mathbf{e}} \cdot D_{\mu} \mathbf{a}_{\nu}=0$.

Several factors simplify the process of extracting the quadratic terms in the action. Because $D_{\mu} \boldsymbol{\Phi}, \mathbf{F}_{r \mu}$ and $\mathbf{F}_{t \mu}$ all vanish for the unperturbed solution, terms containing the product of a metric perturbation and a matter perturbation can only arise from the $\mathbf{F}_{\theta \phi} \cdot \mathbf{F}^{\theta \phi}$ term in $\mathcal{L}_{\text {Matter }}$; it is easy to see that the only matter field that can enter here is $c_{\mu}$. Further, the cross terms between $\mathbf{a}_{\mu}$ and $c_{\nu}$, between $\mathbf{a}_{\mu}$ and $\delta h$, and between $c_{\mu}$ and $\delta h$ all vanish. The result is that the quadratic part of the action may be decomposed as

$$
S_{q u a d}\left(\mathbf{a}_{\mu}, c_{\nu}, \delta \boldsymbol{\Phi}, \delta g_{\mu \nu}\right)=S_{1}\left(c_{\mu}, \delta g_{\mu \nu}\right)+S_{2}(\delta \boldsymbol{\Phi})+S_{3}\left(\mathbf{a}_{\mu}\right)
$$


Since $c_{\mu}$ is the component of the fluctuation lying in the unbroken $U(1)$ subgroup, $S_{1}$ describes an essentially Abelian problem; we therefore do not expect it to contain any unstable modes. Similarly, $S_{2}$ is simply the action for a neutral scalar field in a curved Reissner-Nordström background, and easily shown to give no instabilities.

This leaves us with

$$
\begin{gathered}
S_{3}=\int d^{4} x r^{2} \sin \theta\left[-\frac{1}{4}\left(D_{\mu} \mathbf{a}_{\nu}-D_{\nu} \mathbf{a}_{\mu}\right) \cdot\left(D^{\mu} \mathbf{a}^{\nu}-D^{\nu} \mathbf{a}^{\mu}\right)\right. \\
\left.+\frac{1}{2} e^{2} v^{2} \mathbf{a}_{\mu} \cdot \mathbf{a}^{\mu}+e \mathbf{F}_{\theta \phi} \cdot \mathbf{a}^{\theta} \times \mathbf{a}^{\phi}\right]
\end{gathered}
$$

where indices are understood to be raised by unperturbed metric and $\mathbf{F}_{\theta \phi}$ is the unperturbed magnetic field. Note first that stability would be manifest if it were not for the presence of the last term in the integrand. Indeed, the instability of the $n=1$ solution sets in as soon as this driving term can be greater in magnitude than the mass terms for $\mathbf{a}_{\theta}$ and $\mathbf{a}_{\phi}$ just outside the horizon. With the aid of the inequality

$$
\left|e \mathbf{F}_{\theta \phi} \cdot \mathbf{a}^{\theta} \times \mathbf{a}^{\phi}\right|=\frac{n}{r^{4} \sin \theta}\left|\hat{\mathbf{e}} \times \mathbf{a}_{\theta} \cdot \mathbf{a}_{\phi}\right| \leq \frac{n}{r^{4} \sin \theta}\left|\mathbf{a}_{\theta}\right|\left|\mathbf{a}_{\phi}\right|
$$

it is easily shown that the driving term cannot be dominant, and thus stability is assured, if $r_{H}>\sqrt{n} /(e v)$. Conversely, exponentially growing solutions can be constructed whenever $r_{H}<c \sqrt{n} /(e v)$. An explicit example, which can be verified by substitution into the field equations derived from $S_{3}$, is given by $\mathbf{a}_{t}=\mathbf{a}_{r}=0$ and

$$
\begin{aligned}
& \mathbf{a}_{\theta}=u_{n}(r, t) \sin ^{n-1} \theta \partial_{\theta} \hat{\mathbf{e}} \times \hat{\mathbf{e}} \\
& \mathbf{a}_{\phi}=u_{n}(r, t) \sin ^{n} \theta \partial_{\theta} \hat{\mathbf{e}}
\end{aligned}
$$

where $u_{n}(r, t)$ satisfies Eq. (18), but with $e^{2} v^{2}$ replaced by $n e^{2} v^{2}$ in the potential $U(x)$. As expected, this solution is not spherically symmetric; under rotation, it transforms into other linearly independent solutions. Using Eq. (3), we can see that this instability is present whenever $n \lesssim\left(M_{P} / v\right)^{2}$ and

$$
M<M_{i n s t}=\frac{\sqrt{n} c M_{P}^{2}}{2 e v}+\frac{2 \pi n^{3 / 2} v}{c e}
$$

Some physical understanding of the $n$-dependence of this result can be obtained by returning to the flat space picture of a core region of radius $R$ containing nontrivial Higgs 
and charged boson fields, with only a Coulomb magnetic field extending beyond the core. A variational argument shows that the value of $R$ which minimizes the energy is proportional to $\sqrt{n}$.

This instability has significant implications for the evolution of a magnetically charged black hole. A Reissner-Nordström black hole will lose mass through the emission of Hawking radiation [7]. In the absence of the classical instability, this process would eventually turn off as $M$ approached $M_{c r i t}$, where the Hawking temperature

$$
T_{H}=\frac{M_{P}^{2}}{2 \pi} \frac{\sqrt{M^{2}-M_{c r i t}^{2}}}{\left(M+\sqrt{M^{2}-M_{c r i t}^{2}}\right)^{2}}
$$

vanishes, unless it had lost its magnetic charge in the meantime. Such a discharge could be accomplished by the production of monopole-antimonopole pairs in the strong magnetic field outside the horizon, with one particle falling into the hole and the other moving out to spatial infinity [8]. Pair production of monopoles with magnetic charge $1 / e$ becomes significant only in magnetic fields of magnitude $e M_{m o n}^{2}[9]$. The field at the horizon of a hole with charge $n / e$ is this large only if $M \lesssim M_{\text {pair }} \sim \sqrt{n} M_{P}^{2} / v$ [10]. Since this is a factor of $e$ smaller than $M_{\text {inst }}$, pair production is significant only for black holes which are already classically unstable [11].

The classical instability changes this scenario. Consider first the case of a hole with unit magnetic charge. Thus, suppose that a single magnetic monopole falls into a large neutral black hole, which eventually settles down to a Reissner-Nordström solution. The hole begins to lose mass through the Hawking process. As the mass falls below $M_{\text {inst }}$ and the horizon contracts within the sphere $r=c(e v)^{-1}$, the instability causes nontrivial matter fields to begin to outside the horizon. The black hole is now described by a solution of the type found in Ref. 4. Its horizon continues to contract, revealing more and more of a monopole core. Its temperature, like that of a Schwarzschild black hole, increases monotonically. While the question of its ultimate fate cannot be settled within the semiclassical approximation, the answer will be the same as for a Schwarzschild black hole. If the latter can in fact evaporate completely, then so can our black hole. When it does so, it leaves behind a monopole identical to the one which had fallen in long before. 
This picture is modified slightly if $n>1$. Because the unstable modes are not spherically symmetric, the matter fields which emerge when $M$ falls below $M_{\text {inst }}$ do not form a uniform shell, but are instead localized about isolated points on the horizon. A plausible guess is that as the horizon contracts these grow into lumps which can eventually break off as unit monopoles, thus reducing the magnetic charge of the hole. Eventually only a single charge is left, and the evolution proceeds as described above.

Furthermore, if $M>M_{\text {crit }}$ and $n>\left(M_{P} / v\right)^{2}$, stability is assured since $r_{H}>\sqrt{n} / e v$. Thus, a black hole could be stabilized by endowing it with a sufficiently large magnetic charge. However, this stabilization is not absolute. Pair production, although strongly suppressed, is not quite forbidden. Eventually, enough of the magnetic charge will have been emitted for the monopole instability to emerge.

While these results have been obtained in the context of an $S U(2)$ gauge theory, they clearly can be extended to other gauge theories containing magnetic monopoles. In some theories with two stages of symmetry breaking it is possible to have more than one variety of monopole; e.g. a heavy singly- charged monopole and a somewhat lighter (and spatially larger) doubly-charged one [12]. In such theories the Reissner-Nordström solutions of higher charge presumably become unstable when their horizon is comparable to the the size of the lighter monopole, with the singly charged solution remaining stable until it has shrunk to the size of the heavier one.

One can also obtain magnetic monopole solutions in Kaluza-Klein models [13]. The question of whether these lead to similar instabilities is an interesting one, but is beyond the scope of this letter.

Thus, the effect we have found leads to a remarkable new possibility for the evaporation of a black hole carrying a conserved magnetic charge. Previously, it had seemed that if such a hole did not somehow lose its charge the Hawking process would terminate before complete evaporation was achieved. We see now that charge conservation need not be a barrier to complete evaporation, and that it is quite possible that a magnetically charged black hole could evaporate completely, leaving in its place a nonsingular magnetic monopole.

We thank Hai Ren for pointing out an error in a previous version of this paper. 


\section{REFERENCES}

1. G. 't Hooft, Nucl. Phys. B79, 276 (1974); A.M. Polyakov, Pis'ma Zh. Eksp. Teor. Fiz. 20, 430 (1974) [JETP Lett. 20, 194 (1974)].

2. F.A. Bais and R.J. Russell, Phys. Rev. D11, 2692 (1975); Y.M. Cho and P.G.O. Freund, Phys. Rev. D12, 1588 (1975).

3. J.A. Frieman and C.T. Hill, SLAC preprint SLAC-PUB-4283 (1987).

4. K. Lee, V.P. Nair and E.J. Weinberg, Columbia and Fermilab preprint CU-TP539/FERMILAB-Pub-91/312-A\&T (1991).

5. It is curious, however, that while the radius and the precise form of the monopole core vary somewhat with the couplings in the Higgs potential, the value of $r_{H}$ at which instability occurs does not.

6. E.J. Weinberg and A.H. Guth, Phys. Rev. D14, 1660 (1976).

7. S.W. Hawking, Commun. Math. Phys. 43, 199 (1975).

8. The discharge of electrically charged black holes is discussed in G.W. Gibbons, Commun. Math. Phys. 44, 245 (1975).

9. I.K. Affleck and N.S. Manton, Nucl. Phys. B194, 38 (1982)

10. W.A. Hiscock, Phys. Rev. Lett. 50, 1734 (1983).

11. In conventional units, with $\hbar$ not set equal to unity, $M_{\text {pair }} / M_{\text {inst }}$ is of order $\hbar^{1 / 2}$, reflecting the quantum mechanical nature of pair production as opposed to the classical nature of the instability.

12. G. Lazarides and Q. Shafi, Phys. Lett. 94B, 149 (1980); G. Lazarides, M. Magg, and Q. Shafi, Phys. Lett. 97B, 87 (1980).

13. D.J. Gross and M.J. Perry, Nucl. Phys. B226, 29 (1983); R.D. Sorkin, Phys. Rev. Lett. 51, 87 (1983). 